\title{
Valoración de la potencia anaeróbica en taekwondo. Una revisión sistemática
}

\section{Evaluation of anaerobic power in taekwondo. A systematic review}

\author{
Julio Alberto Aponte-Cáceres (iD; Daniel Enrique Segura Caicedo² (iD
}

\author{
1Universidad Incca de Colombia; j.aac98@hotmail.com \\ ²Universidad de Ciencias Aplicadas y Ambientales U.D.C.A; dasegura@udca.edu.co
}

Cómo citar: Aponte-Cáceres J.A.; Segura-Caicedo, D.E. 2022. Valoración de la potencia anaeróbica en taekwondo. Una revisión sistemática. Rev. Digit. Act. Fis. Deport. 8(1):e1967. http://doi.org/10.31910/rdafd.v8.n1.2022.1967

Artículo de acceso abierto publicado por Revista Digital: Actividad Física y Deporte, bajo una licencia Creative Commons CC BY-NC 4.0

Publicación oficial de la Universidad de Ciencias Aplicadas y Ambientales U.D.C.A, Institución de Educación Superior Acreditada de Alta Calidad por el Ministerio de Educación Nacional.

Recibido: 23 de junio de 2021 Aceptado: 23 de septiembre de 2021 Editado por: Álvaro José Gracia Díaz

\section{RESUMEN}

Introducción: El taekwondo es un deporte de combate, en donde se generan altas exigencias del metabolismo aeróbico y anaeróbico, prevaleciendo la potencia anaeróbica, debido a las acciones de corta duración y alta intensidad ( 1 a 5 segundos), que se presentan en los combates, seguidos de periodos largos de no lucha, con una relación media entre 1:2 y 1:7. Objetivo: Investigar cuáles son los métodos y protocolos utilizados para valorar la potencia anaeróbica en el taekwondo y determinar cuál de estos es el más apropiado. Metodología: La especificidad de la investigación requirió que se priorizara la búsqueda de artículos en las bases de datos ScienceDirect, Taylor \& Francis, Scopus y Ebsco, utilizando palabras claves: valoración, taekwondo y potencia anaeróbica. Resultados: Se identificaron varios métodos de valoración de la potencia anaeróbica en el taekwondo, hallando el Wingate Test, un método denominado Gold Estándar y usado durante años, para dicha valoración y encontrando varias pruebas específicas de Taekwondo recientes, que han surgido a partir de la necesidad de una valoración más específica y objetiva dentro del Taekwondo: FSKT (Fast Speed Kick Test),
FSKTmult (Fast Speed Kick Test Mult), TAT (Taekwondo Anaerobic Test), TSAT (Taekwondo Specific Anaerobic Test) y TAIKT (Taekwondo Anaerobic Intermittent Kick Test). Conclusiones: TAIKT, FSKT y FSKTmult son las pruebas más confiables y de mayor acceso para entrenadores y centros deportivos, que requieran valorar la potencia anaeróbica de taekwondo, facilitando el control y el seguimiento, en busca de mejorar el rendimiento de los deportistas y llegar a obtener altos logros.

Palabras clave: Valoración; Potencia anaeróbica; Taekwondo.

\section{ABSTRACT}

Introduction: Taekwondo is a combat sport, where high demands on aerobic and anaerobic metabolism are generated, with anaerobic power prevailing due to the short, high-intensity actions (1-5 seconds) that occur during combat, followed by long periods of does not fight with an average ratio between 1: 2 and 1: 7. Objective: To know the existing methods and protocols to assess anaerobic power in this sport and determine which of these is the most appropriate. 
Methodology: The specificity of the research required that the search for articles in databases such as: ScienceDirect, Taylor \& Francis, Scopus and Ebsco be prioritized, using keywords such as: valuation, taekwondo and anaerobic power. Results: As a result of the exhaustive review, it was possible to identify various methods of assessing anaerobic power in taekwondo, mainly finding the Wingate Test, a method known as Gold Standard and used for years for such assessment and finding several relatively new specific Taekwondo tests. that have arisen from the need for a much more specific and objective assessment within Taekwondo, such as: FSKT (Fast Speed Kick Test), FSKTmult (Fast Speed Kick Test Mult), TAT (Taekwondo Anaerbic Test), TSAT (Taekwondo Specific Anaerobic Test), and TAIKT (Taekwondo Intermittent Kick Anaerobic Test). Conclusions: That the TAIKT, FSKT and FSKTmult tests are the most reliable and widely accessible tests for coaches and sports centers that need to assess the anaerobic power of taekwondo, facilitating the control of said capacity, as well as its monitoring in order to improve the performance of athletes and achieve high achievements.

Keywords: Evaluation; Anaerobic power; Taekwondo.

\section{INTRODUCCIÓN}

El taekwondo es un deporte de combate, en donde se generan altas exigencias del metabolismo aeróbico y anaeróbico, prevaleciendo la potencia anaeróbica, debido a las cortas acciones de alta intensidad (1 a 5 segundos), que se presentan durante los combates, seguidos de periodos largos de no lucha, con una relación media entre $1: 2$ y 1:7, alcanzado frecuencias cardiacas pico del $90 \%$, de la FCmáx. y concentraciones de lactato sanguíneo, entre 7,0 a 12,2 mmol.l (Cardozo et al. 2017), es decir, la potencia anaeróbica resulta una capacidad determinante en el taekwondo, siendo vital realizar una valoración y un control de dicha capacidad.

La potencia anaeróbica es la propiedad física orgánica que posibilita la realización de un esfuerzo de alta intensidad en un tiempo determinado. Puede ser aláctica, si compromete las reservas de fosfógeno (ATP-PC), cuando el esfuerzo no sobrepasa los 10 a 12 segundos o láctica, si se activa el mecanismo glucolítico con producción de ácido láctico, por encima de los valores normales (9 a $16 \mathrm{mg} / 100 \mathrm{ml}$ de sangre), sin sobrepasar los tres minutos de esfuerzo
(Guzmán Palacio \& Jiménez Trujillo, 2013; Hornillos Baz, 2010).

La importancia de evaluaryde controlarlascapacidades físicas determinantes de un deporte es primordial para la planificación, que permita incrementar el rendimiento deportivo, facilitando conocer el estado de los deportistas y efectuar el seguimiento óptimo. Por esta razón, la presente investigación tiene como objetivo realizar una revisión de la literatura científica, sobre cuáles son los métodos y protocolos utilizados para valorar la potencia anaeróbica en el taekwondo y determinar cuál de estos es el más apropiado.

\section{MATERIALES Y MÉTODOS}

Para la presente revisión sistemática, se indagó sobre los principales métodos y protocolos utilizados para valorar la potencia anaeróbica en el taekwondo. Como parte inicial del proceso, se realizó una búsqueda exhaustiva de la literatura científica, utilizando las bases de datos ScienceDirect, Taylor \& Francis, Scopus y Ebsco, anteponiendo las palabras claves: valoración, taekwondo, potencia anaeróbica y aplicando ecuaciones de búsqueda en idioma inglés: "Taekwondo and Anaerobic Power", sumando a esta ecuación "Assessment".

La búsqueda inicial permitió encontrar 182 artículos, que fueron filtrados, teniendo en cuenta los criterios de inclusión y de exclusión:

- Publicaciones en revistas científicas escritas en idioma inglés o español.

- Población de estudio en deportistas de taekwondo.

- Artículos publicados posterior al 2011.

- Artículos full Access que permitieran la descarga completa.

- Artículos que en el contenido no especifican un método o protocolo de evaluación o control de la potencia anaeróbica dentro de la disciplina del taekwondo.

- Exclusión de artículos repetidos.

Finalizando esta revisión de criterios de inclusión y de exclusión, se descartaron 3 artículos, que fue imposible acceder al texto completo y, por tal motivo, no se pudo obtener la información completa de estos. También, se descartaron 4 artículos científicos repetidos dentro de las bases de datos y, por último, fueron excluidos 2, que no cumplieron con el año de publicación, siendo artículos publicados antes del 


\section{Revista Digital: Actividad Física y Deporte}

2011. De esta forma, la presente revisión sistemática está basada en el estudio a profundidad de un total de 23 artículos (Figura 1), que cumplieron todos los criterios de inclusión y de exclusión y fueron aptos para aportar, de manera determinante en la investigación.
Sumado a esto, se realizó una validación de la calidad de los artículos, a través de una escala dicotómica, tomando como referencia la matriz propuesta por Law et al. (1998), que dio como resultado, una calidad superior al $75 \%$, como indicador de confiabilidad.

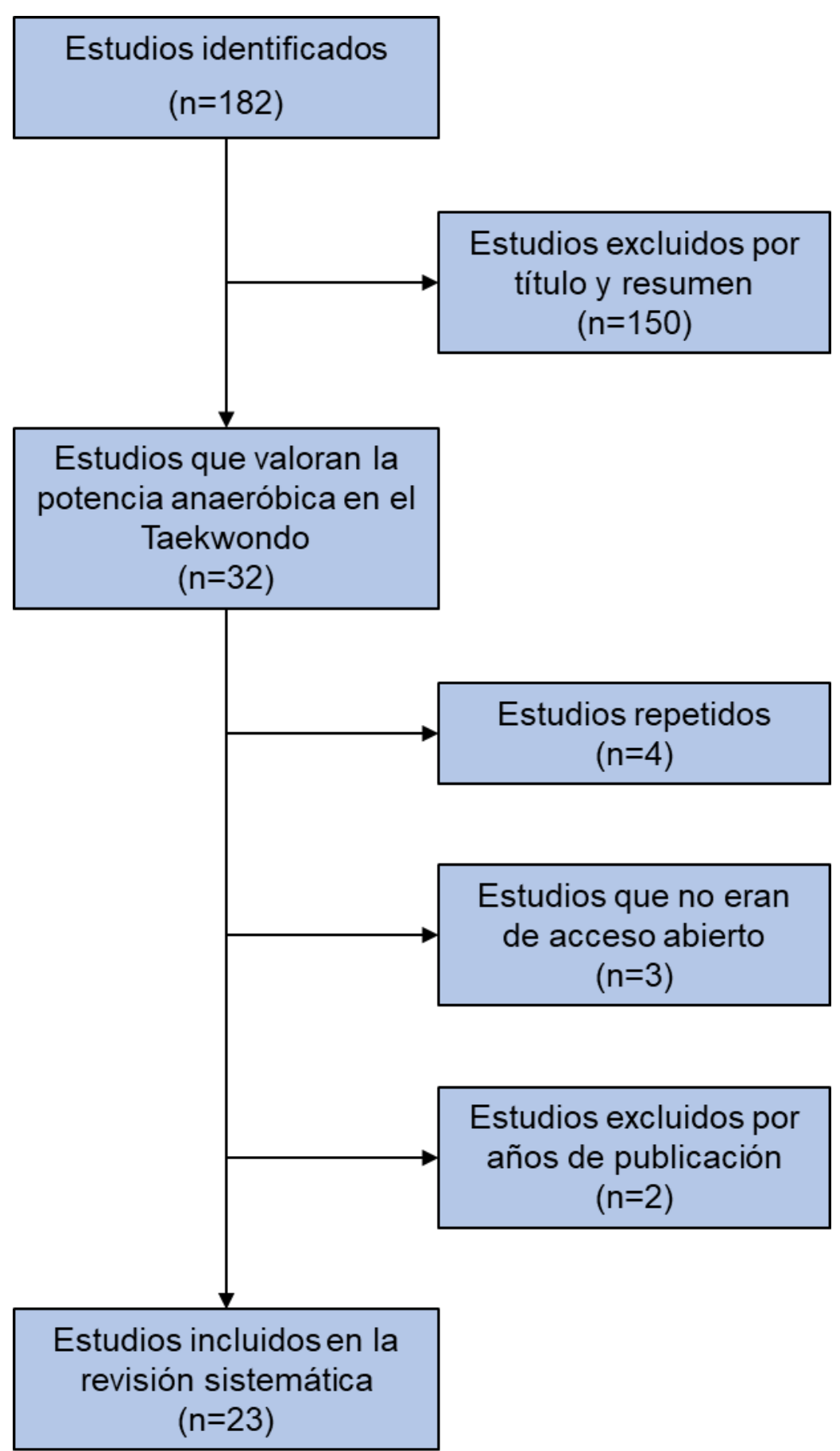

Figura 1. Procedimiento de inclusión y de exclusión de bibliografía, relacionada con la potencia anaeróbica en taekwondo. 


\section{RESULTADOS Y DISCUSIÓN}

Tras realizar una revisión exhaustiva, se identificaron diferentes métodos empleados para la medición de la potencia anaeróbica en los atletas de taekwondo. A continuación, se muestran las principales características de cada uno.

Fast Speed Kick Test (FSKT). La prueba Fast Speed Kick Test (FSKT) es un test específico de taekwondo, que mide la frecuencia de pateo, en un tiempo determinado. Consiste en realizar el mayor número de patadas posibles con la Técnica Bandal Chagui, alternando la pierna derecha e izquierda, durante 10 segundos, técnica usada, frecuentemente, dentro de los combates, por su alta efectividad, facilidad y velocidad, a la hora de marcar los puntos. Para dicha prueba, cada atleta, se debe ubicar frente a un saco, equipado con un protector de pecho de taekwondo; se utilizan dos evaluadores, uno se encarga de cronometrar el tiempo de las cinco series, mientras que el otro, de sostener el saco y contar el número de patadas ejecutadas correctamente (Da Silva Santos \& Franchini, 2018; Da Silva Santos et al. 2018; Da Silva Santos et al. 2019; Aravena Tapia et al. 2020; Da Silva Santos et al. 2020; Castro-Gatrido et al. 2020).

Fast Speed Kick Test Mult (FSKTmult). La prueba Fast Speed Kick Test Mult (FSKTmult) es un test específico de taekwondo que, al igual que el FSKT, mide la frecuencia de pateo en un tiempo determinado. El procedimiento es el mismo del FSKT, pero ejecutando cinco series un intervalo de descanso de 10 segundos entre serie y determinando el rendimiento con el número de patadas de cada set; el total de patadas durante las cinco series y el índice de fatiga, que se establece con la ecuación 1 (Da Silva Santos et al. 2019):

Kick decrement index $(\%)=1-\frac{(\text { FSKT1+FSKT2 }+ \text { FSKT3 }+ \text { FSKT5 })}{\text { Best FSKT Set X Number of sets }}$

Taekwondo Anaerobic Intermittent Kick Test (TAIKT). La prueba de patada intermitente anaeróbica de taekwondo (TAIKT) es un protocolo diseñado específicamente para este deporte. Una de sus ventajas es que permite medir y calificar la capacidad anaeróbica mediante la patada "Bandal-Chagui" que, como ya se señaló, es una de las principales empleadas en los combates de este deporte. Después de una señal auditiva, el deportista debe realizar el máximo número de patadas "Bandal-Chagui", a un saco de boxeo, que tiene un protector corporal electrónico. Además, debe alternar las dos piernas durante 5 segundos y lo repite 6 veces (series), teniendo un tiempo de 10 segundos de recuperación activa entre serie; debido al protector corporal electrónico, el número de patadas ejecutadas por el deportista, se proyecta en un computador después de cada serie (Tayech et al. 2019).

Este protocolo busca calificar y evaluar la potencia anaeróbica específica, teniendo en cuenta el máximo número de patadas, la media de patadas y la frecuencia absoluta del número de patadas ejecutadas por el deportista (Sarshin et al. 2021).

Taekwondo Anaerobic Test (TAT). La prueba Taekwondo Anaerobic Test (TAT) es un test usado para medir la potencia anaeróbica en el taekwondo. Consiste en realizar el mayor número de patadas de la Técnica Bandal Chagui a la mayor intensidad posible, alternando la pierna de pateo a una altura media, marcada en el objeto. Para la medición, se coloca un acelerómetro triaxial sobre el tobillo dominante de atleta y se utilizan tres amplificadores de señal (De Sousa Fortes et al. 2016). Así, se miden el número de ciclos realizados, el tiempo medio de los ciclos de patadas, el mejor tiempo entre ciclos y el índice de fatiga, que se establece con la ecuación 2 (Sant'Ana et al. 2014):

$\mathrm{FI}$ por tiempo= $(\mathrm{LC}-\mathrm{IC} / \mathrm{IC}) \times 100$; FI por impacto: (IC-LC/IC) X 100 ecuación 2

\section{Donde:}

$\mathrm{FI}=$ índice de fatiga

$\mathrm{LC}=$ tiempo promedio de pateo/impacto de al menos el $20 \%$ de los ciclos

$\mathrm{IC}=$ tiempo promedio de pateo/impacto en el $20 \%$ de los ciclos iniciales

A partir de este criterio de medida, se puede calcular o evaluar el número de ciclos de patadas, el mejor tiempo y el tiempo medio de las patadas. También, se pudieron medir los impactos de las patadas durante los $30 \mathrm{~s}$, el impacto medio y el impacto más alto. Todos los datos de impacto fueron expresados en gravedad y los datos de TAT, se expresaron por el número total de ciclos de patadas (100 \%) y valores medios cada (10 \%) (Sant'Ana et al. 2014).

Taekwondo Specific Anaerobic Test (TSAT). La prueba Taekwondo Specific Anaerobic Test (TSAT) 
es un test que mide diferentes variables, entre las cuales, se encuentra la potencia anaeróbica media, potencia anaeróbica media relativa, el índice de fatiga y la capacidad anaeróbica. La prueba consiste en realizar la técnica de Pateo Bandal Chagui a la máxima velocidad y potencia posible, durante 30 segundos, alternando pierna derecha e izquierda. El deportista, con esta técnica, debe impactar un instrumento de medición, que es sostenido por un evaluador y tiene un sensor que evalúa la fuerza del impacto y el número de ejecuciones realizadas (Rocha et al. 2016).

Wingate test. Esta prueba, se realiza en un cicloergómetro, en donde el atleta debe pedalear a máxima velocidad, durante 30 segundos, frente a una resistencia de $0,075 \mathrm{~kg} / \mathrm{kg}$ (cicloergómetro Monark) y mediante programas y el mismo cicloergómetro, el resultado se va a ver reflejado en un computador. Esta prueba permite valorar la capacidad anaeróbica de una manera muy completa, teniendo en cuenta el tiempo en el que el atleta alcanza la potencia máxima y cómo la sostiene o disminuye, según transcurre el tiempo. La mayoría de los estudios señalan la importancia de una estimulación verbal hacia el deportista, para que intente llegar a la velocidad máxima en el menor tiempo posible y el esfuerzo sea del $100 \%$ (Morales et al. 2015; Mine \& Yağmur, 2016; Rocha et al. 2016; Bakońska-Pacón et al. 2017; Boraczyński et al. 2017; Hanson et al. 2018; Kwon et al. 2019; Taskin \& Akkoyunlu, 2020; Tayech et al. 2020).

Teniendo en cuenta, los diferentes hallazgos de la presente revisión sistemática, se identifica una gran variedad de métodos y protocolos para la evaluación de la potencia anaeróbica en el taekwondo y considerando que el objetivo fue revisar en la literatura científica cómo son esos métodos y protocolos utilizados, para valorar dicha capacidad en este deporte y determinar cuál de estos es el más apropiado, se analizaron los diferentes hallazgos, determinando el uso de las diferentes pruebas y su influencia en el rendimiento de atletas de taekwondo.

La prueba Wingate test, en la mayoría de los estudios analizados en esta revisión, se identifica como la prueba reina para calificar y medir la capacidad anaeróbica de los miembros inferiores en el deporte, permitiendo hacer comparaciones con otros deportes, debido a su alta confiabilidad. Según Tayech et al. (2019), esta prueba es considerada como la "Gold standard" para la valoración de la potencia anaeróbica máxima y media (Taskin \& Akkoyunlu, 2020).
Un estudio realizado por Mine \& Yağmur (2016), analiza los efectos de la potencia anaeróbica, a través del Wingate test, sobre la prueba de velocidad máxima de 5 metros, en 30 deportistas de taekwondo femenino, de la selección nacional de Turquía, analizando las variables de potencia anaeróbica máxima y potencia anaeróbica media, concluyendo, que hay una correlación positiva entre el aumento de la potencia anaeróbica y el aumento del rendimiento en la velocidad máxima de las mujeres deportistas de taekwondo. Otro estudio realizado por Taskin \& Akkoyunlu (2020), utilizó el Wingate test, para evaluar la potencia anaeróbica de 16 hombres de la selección nacional de Turquía, determinando su influencia en la rapidez y agilidad de los deportistas, destacando, que la potencia anaeróbica máxima es un indicador importante para el rendimiento de la agilidad y concluye que hay una correlación significante entre el aumento de la potencia anaeróbica medida, la rapidez y la agilidad.

Morales et al. (2015) analizaron la influencia del uso de protector bucal sobre la potencia anaeróbica, de 28 deportistas masculinos de taekwondo; para esto, los deportistas realizaron entre 3 y 4 entrenamientos por semana y utilizaron el Wingate test con y sin protector bucal, para evaluar dicha capacidad. En los resultados, se evidenciaron diferencias significativas en todas las variables de la prueba y concluyeron, que los deportistas de taekwondo que entrenan con protector bucal, alcanzaron rendimientos más altos en la potencia anaeróbica y obtuvieron una menor acumulación de lactato en sangre. Por otro lado, Hanson et al. (2018) determinaron la influencia del protector bucal (Under Armour) sobre la capacidad anaeróbica, en comparación con otros protectores bucales e, incluso, sin protector bucal; para esto, utilizaron el Wingate test, en 17 deportistas de élite de taekwondo, hallando, en este caso, que no se encuentran diferencias significativas entre el uso del bucal (Under Armour) y otras marcas o sin dicho protector.

Bakońska-Pacón et al. (2017) evaluaron la influencia de un programa de pérdida de peso gradual de 6 semanas sobre la potencia anaeróbica, la composición corporal y la fuerza muscular, en 18 deportistas de élite de la selección nacional de Polonia. Para evaluar la potencia anaeróbica, se empleó el Wingate test, afirmando que es la más utilizada para valorar esta capacidad y realizando un pre-test y un post-test, para analizar los resultados, concluyendo, que no hubo 
una diferencia significativa entre las dos pruebas y afirmando que el programa de 6 semanas de pérdida de peso gradual, no afectaba, de manera negativa, en la potencia anaeróbica de los deportistas.

Una revisión sistemática realizada por Vasconcelos et al. (2020), analizan los efectos del entrenamiento de intervalos de alta intensidad (HIIT), en los deportes de combate, destacando la prueba Wingate test, como la más utilizada, para medir el perfil anaeróbico de los deportistas y concluyeron que, con este método de entrenamiento, se genera una mayor potencia anaeróbica, mejorando el rendimiento de los atletas.

Kwon et al. (2019), en un estudio, afirman que la prueba Wingate test es un método óptimo para la evaluación y el análisis de la potencia anaeróbica; sin embargo, reconocen las principales limitantes de este método, siendo el alto costo en los equipos e inspectores capacitados para utilizarlos, así como el laboratorio o espacio específico adecuado, para realizar dicha evaluación y, por último, la falta de especificidad dentro del taekwondo.

El Wingate Test, también ha sido utilizada en otros deportes, por su alta confiabilidad, independiente del deporte. Stauffer et al. (2015) aplicaron esta prueba para valorar la potencia anaeróbica en atletas de baloncesto; estos autores señalan que la prueba se considera el instrumento más válido y confiable para evaluar la potencia máxima y la capacidad anaeróbica. Además, afirman que, para evaluar adecuadamente las características anaeróbicas en deportistas, se deben emplear actividades específicas del deporte, haciendo referencia a que hace falta documentar y validar las pruebas específicas que existen, hoy en día, para valorar la potencia anaeróbica en el baloncesto, es por esto, que recurren a la prueba Wingate, como guía principal, para valorar esta capacidad. Por otro lado, Cieśliński et al. (2021) utilizaron esta prueba para valorar la capacidad anaeróbica de los músculos de las extremidades inferiores y superiores de deportistas luchadores de estilo libre, confirmando la fiabilidad de esta prueba.

Distintos estudios han buscado la especificidad en la evaluación de la potencia anaeróbica dentro del taekwondo, hallando diferentes métodos específicos, que podrían cumplir dicho fin; uno de ellos es el TSAT. Rocha et al. (2016) realizan un estudio, en donde se evidencia la efectividad de la prueba TSAT, para evaluar la potencia anaeróbica, en atletas élite de taekwondo.
En dicho estudio, se aplicó la prueba en dos sesiones distintas, con diferencia de una semana, para evaluar la confiabilidad y se determinó que los resultados de potencia anaeróbica media y potencia anaeróbica relativa tuvieron una diferencia entre pequeña y media, observando una tendencia de valores más altos hacia la segunda prueba, mientras que, en las variables de capacidad anaeróbica e índice de fatiga, no se encontraron diferencias estadísticamente significativas.

Estos autores, también compararon la prueba TSAT con el CMJ y el Wingate test, encontrando una correlación significativa entre el CMJ y la potencia anaeróbica media en la prueba TSAT, así como un mayor número de patadas realizadas. Por otro lado, las variables entre el Wingate test y la prueba TSAT, estaban significativamente correlacionadas, siendo el índice de fatiga la correlación más alta, concluyendo, que ambas pruebas, para medir la potencia anaeróbica, proporcionan una coherencia similar.

Sant'Ana et al. (2014) proponen otro método de evaluación anaeróbica para taekwondo, denominado TAT, en donde se utiliza la Bandal Chagui, durante 30s (basados en la prueba de Wingate), tiempo adecuado para analizar los sustratos energéticos de ATP-PC y Glucolisis anaeróbica y las concentraciones de lactato, variables que indican dependencia del sistema anaeróbico. En este estudio, 10 atletas de taekwondo realizan la prueba, en donde se mide el pico de impacto generado por la pierna dominante con el acelerómetro, con el fin de medir el tiempo empleado en cada ciclo de patadas, definido como el intervalo de tiempo entre dos patadas consecutivas con la misma pierna.

Se evidenció que hubo un aumento significativo en el tiempo del ciclo de patadas durante el último $20 \%$ de la prueba, en comparación con el ciclo de patadas del $20 \%$ inicial; el índice de fatiga obtenido de la comparación de estas variables reveló una caída del $11,14 \%$ en el rendimiento, durante los 30 s totales de la prueba. Asimismo, hubo una disminución en la magnitud del impacto del ciclo de patadas durante el último $20 \%$ de la prueba, en comparación con el ciclo de patadas del $20 \%$ inicial; el índice de fatiga obtenido de la comparación de estas variables mostró una caída del 27,69 \% en el impacto, durante los 30 $s$ totales de la prueba. Concluyendo que todos los sujetos que realizaron la prueba tuvieron el mejor tiempo de pateo durante los primeros $10 \mathrm{~s}$, lo que 
se puede comparar con el Wingate Test, en donde la potencia máxima se genera durante los primeros $5 \mathrm{~s}$, que representa la mayor cantidad de energía liberada por el sistema anaeróbico aláctico ATP-PC (potencia anaeróbica aláctica). También, se evidencia una correlación entre el CMJ y el mejor tiempo de pateo, confirmando estos hallazgos, al igual que el lactato en sangre, que tuvo una correlación entre el número de patadas realizadas y un aumento de estas concentraciones de lactato en sangre.

De Sousa Fortes et al. (2016) realizan un estudio con 31 deportistas de taekwondo que compiten a nivel nacional, en Brasil, en donde afirman que dos semanas de puesta a punto, optimizaban la potencia y la capacidad anaeróbica en deportistas de taekwondo y la reducción del volumen de entrenamiento en las semanas previas a una competencia, pueden ayudar a mejorar el rendimiento en esta capacidad; sin embargo, muestran como limitantes dentro del estudio que, al no medir el lactato en sangre después del test TAT, ni la magnitud de motivación, no se puede decir que todos los deportistas hayan realizado dicho test con su máximo esfuerzo, lo cual, podría haber influido en los hallazgos.

Otro método utilizado para medir la potencia anaeróbica dentro del taekwondo es el TAIKT. Tayech et al. (2019) adelantan un estudio para verificar la confiabilidad test-retest y la validez de la prueba específica de taekwondo TAIKT; para esto, 20 atletas del equipo nacional de Túnez, ejecutaron la prueba TAIKT dos veces, en un intervalo de tres semanas, junto con la prueba Running-based Anaerobic Sprint Test (RAST), debido a que tienen protocolos similares y este último es usado para medir el rendimiento anaeróbico, de forma general, al igual que el Wingate Test. Los resultados de la investigación presentaron un índice de confiabilidad excelente (superior a 0,90), entre test y pretest y una correlación entre grande y muy grande, entre las dos pruebas, concluyendo, que la prueba TAIKT es una prueba confiable para atletas de taekwondo. Sarshin et al. (2021) confirman la fiabilidad de la prueba TAIKT en un estudio realizado con cuarenta atletas de taekwondo, con más de dos años de experiencia, en competencias de la liga de taekwondo de Irán.

Diferentes autores proponen otro método para evaluar la potencia anaeróbica en el taekwondo. Da Silva Santos \& Franchini (2016) afirman que el FSKT es una nueva herramienta utilizada para evaluar esta capacidad y puede ser un indicador de rendimiento en el taekwondo; dentro de su estudio proponen que los deportistas que obtienen un mejor resultado en esta prueba tienen más posibilidades de realizar movimientos de alta intensidad durante una contienda de taekwondo y aumentar las probabilidades de ganar. Dentro de las conclusiones de este estudio, los autores aseguran la especificidad de la prueba y su sensibilidad para usarse en temporadas competitivas.

Da Silva Santos et al. (2019) afirman que esta prueba presenta un coeficiente de correlación intraclase medio entre test - retest (ICC $=85)$, teniendo una variación de 3,9\%, entre estos; Da Silva Santos et al. (2020) resaltaron también la alta fiabilidad que tiene esta prueba respecto a las variables de frecuencia de pateo durante cada set y la suma de 5 sets junto al índice de disminución de patadas realizadas.

Esta prueba es una herramienta idónea que imita muchas características presentadas dentro de un combate de taekwondo y que puede ser utilizada para monitorear la potencia anaeróbica máxima en los deportistas (Da Silva Santos \& Franchini, 2018). Generalmente, se encuentra una correlación positiva entre una mayor potencia anaeróbica máxima y un mayor número de técnicas realizadas dentro del combate, así como también, existe una correlación positiva con la probabilidad de ganar dicha contienda. También, se encuentran diferencias significativas entre los atletas de diferente nivel competitivo (internacional, nacional y estatal) y el resultado de la prueba FSKT, siendo el nivel internacional, el de mejor rendimiento en dicha prueba y el nivel estatal, en más bajo. Da Silva Santos et al. (2018) afirman algunas limitaciones de esta prueba, concluyendo, que la prueba se puede ver influenciada por la técnica del deportista, así como por la fuerza aplicada en cada una de las técnicas, teniendo en cuenta, que la aplicación de una fuerza mayor en la técnica puede resultar en menor número de repeticiones.

A lo largo de este estudio, se evidenciaron los diferentes métodos y protocolos utilizados para la evaluación de la potencia anaeróbica en el taekwondo, hallando, principalmente, el Wingate Test, un método denominado como Gold Estándar y usado durante años para dicha valoración y encontrando varias pruebas específicas de Taekwondo, relativamente nuevas, que han surgido a partir de la necesidad de una valoración mucho más específica y objetiva dentro del Taekwondo; sin embargo, el presente estudio presenta 
como limitación, la reducida información científica sobre la valoración de la potencia anaeróbica en el taekwondo y la poca población estudiada, dentro de los artículos encontrados. Esta investigación podría ser un punto de partida para próximos estudios con grupos poblacionales mucho más amplios y para dar a conocer a entrenadores y profesionales del deporte los distintos métodos específicos para la valoración de la potencia aeróbica, en el deporte.

\section{CONCLUSIONES}

El objetivo de la presente revisión sistemática era realizar una revisión de la literatura científica sobre cuáles son los métodos y protocolos utilizados para valorar la potencia anaeróbica en el taekwondo y determinar cuál es el más apropiado. En la persecución de este objetivo y como resultado de una exhaustiva revisión bibliográfica fue posible recopilar información pertinente, para identificar los diferentes métodos que existen, en la actualidad, para la valoración de la potencia anaeróbica en el taekwondo.

Se concluye, que la prueba Wingate es la más utilizada; sin embargo, carece de especificidad y se diferencia de una prueba específica en varios aspectos, como, por ejemplo, la posición de aplicación, porque el Wingate Test, se aplica en una posición sedante, mientras que una prueba específica requiere permanecer de pie; el tipo de movimiento, porque el Wingate Test se ejecuta de forma cíclica en un solo set y, por el contrario, las pruebas específicas, se asemejan a un combate de taekwondo, realizando varios sets de alta intensidad, con descansos activos de forma acíclica y, por último, el equipo específico que requiere el Wingate Test (software y cicloergómetro), en cambio varios de las pruebas específicas son simples y de bajo costo (Bridge et al. 2014).

De igual manera, se infiere que hay varias pruebas específicas de taekwondo, para la valoración de la potencia anaeróbica, que podrían aportar de igual forma y con una fiabilidad científica, al igual que el Wingate Test; sin embargo, hay varias pruebas que tienen ciertas limitantes. La prueba Taekwondo Specific Anaerobic Test (TSAT) es una prueba que tiene la misma confiabilidad que el Wingate Test, pero requiere de un instrumento de medición específico, que hace que no sea de fácil aplicación en centros deportivos que carezcan de éste $y$, por otro lado, es una prueba que dura 30s, alejándose de las acciones reales de un combate de taekwondo. La prueba
Taekwondo Anaerobic Test (TAT), también requiere de un instrumento específico (acelerómetro triaxial), para medir las variables establecidas y aunque su correlación con el Wingate Test para valorar la potencia anaeróbica es alta, también sobrepasa el tiempo en el que se realizan las acciones de taekwondo, siendo de 30s, al igual que el TSAT.

La prueba de Patada intermitente anaeróbica de taekwondo (TAIKT) es muy específica en los intervalos de tiempo, semejantes al combate de taekwondo; sin embargo, su única limitación es el uso de protectores electrónicos de taekwondo, que impediría su valoración en centros deportivos, donde no se posean. Por último, la prueba Fast Speed Kick Test (FSKT) y Fast Speed Kick Test Mult (FSKTmult) son pruebas específicas, en donde no se requiere mayor equipamiento que un protector de pecho convencional del deporte o un pao de impacto y su protocolo es específico, dentro de los intervalos de tiempo usados en un combate, concluyendo, que las pruebas TAIKT, FSKT y FSKTmult son las pruebas más confiables y de mayor acceso para entrenadores y centros deportivos, que requieran valorar la potencia anaeróbica de taekwondo, facilitando el control de dicha capacidad, así como su seguimiento, en busca de mejorar el rendimiento de los deportistas y llegar a obtener altos logros.

Conflicto de intereses: Los autores manifiestan o tener ningún conflicto de intereses.

\section{REFERENCIAS}

1. ARAVENA TAPIA, D.E.; ROMÁN BARRERA, V.; DA SILVA SANTOS, J.F.; FRANCHINI, E.; VALDÉSBADILLA, P.; ORIHUELA, P.; HERRERAVALENZUELA, T. 2020. High-intensity interval training improves specific performance in taekwondo athletes. Revista de Artes Marciales Asiáticas. 15(1):4. https://doi.org/10.18002/rama.v15i1.6041

2. BAKOŃSKA-PACÓN, E.; DZIUBEK, W.; FORGIEL, G.; MURAWSKI-CIALOWICZ, E.; WITWITKOWSKI, K. 2017. Effects of gradual weight loss on anaerobic capacity and muscle strength in elite taekwondo ITF athletes. Archives of Budo. 13:117-130.

3. BORACZYŃSKI, M.; BORACZYŃSKI, T.; PODSTAWSKI, R.; LASKIN J.; CHOSZCZ, D.; LIPIŃSKI, A. 2017. 
Relationships between anthropometric features, body composition, and anaerobic alactic power in elite post-pubertal and mature male taekwondo athletes. Human Movement. 18(4):3-12.

https://doi.org/10.1515/humo-2017-0032

4. BRIDGE, C.; SANTOS, J.; CHAABENE, H.; PIETER, W.; FRANCHINI, E. 2014. Perfiles físicos y fisiológicos de los atletas de taekwondo. Sports Med. 44: 713-733.

5. CARDOZO, L.A.; VERA-RIVERA, D.A.; CONDECABEZAS, O.A.; YÁNEZ, C.A. 2017. Aspectos fisiológicos de deportistas elite de taekwondo: una revisión narrativa. Revista Española de Educación Física y Deportes. 418(3):35-46.

6. CASTRO-GATRIDO, N.; VALDERAS-MALDONADO, C.; HERRERA-VALENZUELA, T.; DA SILVA, J.F.; GUZMÁN-MUÑOZ, E.; VÁSQUEZ-GÓMEZ, J.; BRANCO, B.M.; ZAPATA-BASTÍAS, J.; LÓPEZFUENZALIDA, A.; VALDÉS-BADILLA, P. 2020. Effects of post-activation potentiation exercises on kicking frequency, fatigue rate and jump performance in taekwondo athletes: A case study. Retos. 38:679-683. https://doi.org/10.47197/retos.v38i38.76755

7. CIEŚLIŃSKI, I.; GIERCZUK, D.; SADOWSKI, J. 2021. Identification of success factors in elite wrestlers-An exploratory study. PLoS ONE. 16(3):1-13.

https://doi.org/10.1371/journal.pone.0247565

8. DA SILVA SANTOS, J.; FRANCHINI, E. 2016. Is frequency speed of kick test responsive to training? A study with taekwondo athletes. Sport Sciences for Health. 12(3):377-382. https://doi.org/10.1007/s11332-016-0300-2

9. DA SILVA SANTOS, J.E.; LOPES-SILVA, J.P.; LOTURGO, I.; FRANCHINI, E. 2020. Test-retest reliability, sensibility and construct validity of the frequency speed of kick test in male blackbelt taekwondo athletes. Ido Movement for Culture. Journal of Martial Arts Anthropology. 20(3):38-46.

https://doi.org/10.14589/ido.20.3.6
10. DA SILVA SANTOS, J.F.; FRANCHINI, E. 2018. Frequency speed of kick test performance comparison between female taekwondo athletes of different competitive levels. Journal of Strength and Conditioning Research. 32(10):2934-2938.

https://doi.org/10.1519/JSC.0000000000002552

11. DA SILVA SANTOS, J.F.; HERRERA-VALENZUELA, T.; FRANCHINI, E. 2019. Establishing frequency speed of kick test classificatory tables in male and female taekwondo athletes. Kinesiology. 51(2):213-218.

https://doi.org/10.26582/k.51.2.12

12. DA SILVA SANTOS, J.F.; LOTURCO, I.; FRANCHINI, E. 2018. Relationship between frequency speed of kick test performance, optimal load, and anthropometric variables in blackbelt taekwondo athletes. Ido Movement for Culture. Journal of Martial Arts Anthropology. 18(1):39-44.

https://doi.org/10.14589/ido.18.1.6

13. DE SOUSA FORTES, L.D.; VASCONCELOS, G.C.; MEIRELES DOS SANTOS, T.; PINHEIRO PAES, P.; MACEDO VIANNA, J.; PÉREZ, A.J. 2016. Effect of tapering on anaerobic power and capacity of tae-kwon-do athletes. Rev Bras Cineantropom Desempenho Hum. 19(2):287-296. https:// doi.org/10.1590/1980-0037.2017v19n2p224

14. GUZMÁN PALACIO, J.E.; JIMÉNEZ TRUJILLO, J.T. 2013. Efectos de un plan de entrenamiento de resistencia sobre el VO2 máximo, la frecuencia cardíaca de reposo y los índices de recuperación en futbolistas juveniles. Revista de Educación Física. 2(4):33-91.

15. HANSON, N.J.; LOTHIAN, D.D.; MILLER, C.L.; MICHAEL, T.J.; MILLER, M. 2018. Overthe-counter performance enhancing mouthguards are unable to decrease blood lactate and improve power output during a Wingate anaerobic test (WAnT). Journal of Exercise Science and Fitness. 16(3):83-86. https://doi.org/10.1016/j.jesf.2018.07.001

16. HORNILLOS BAZ, I. 2010. La capacidad acelerativa en el deporte. Cultura, Ciencia y Deporte. 5(15):12-14. 
17. KWON, C.; LEE, S.; PARK, J.; JOHNSON, J.A. 2019. An estimation model for anaerobic power of taekwondo athletes based on field tests. Ido Movement for Culture. Journal of Martial Arts Anthropology 19(1):34-50.

https://doi.org/10.14589/ido.19.1.4

18. LAW, M.; STEWART, D.; POLLOCK, N.; LETTS, L. BOSCH, J.; WESTMORLAND, M. 1998. Critical review form- Quantitative Studies. 1-3p.

19. MINE, T.; YAĞMUR, A. 2016. Effect of anaerobic power on quickness in women national taekwondo athletes. Ovidius University Annals, Series Physical Education \& Sport/ Science, Movement \& Health. 16(2):701-705.

20. MORALES, J.; BUSCÀ, B.; SOLANA-TRAMUNT, M.; MIRÓ, A. 2015. Acute effects of jaw clenching using a customized mouthguard on anaerobic ability and ventilatory flows. Human Movement Science. 44:270-276. https://doi.org/10.1016/j.humov.2015.09.008

21. ROCHA, F.; LOURO, H.; MATÍAS, R.; COSTA, A. 2016. Anaerobic fitness assessment in taekwondo athletes. A new perspective. Motricidade. 12(2):127-139. https://doi.org/10.6063/motricidade.8954

22. SANT'ANA, J.; DIEFENTHAELER, F.; DAL PUPO, J.; DETANICO, D.; GUILHERME, L.; SANTOS, S.G. 2014. Anaerobic evaluation of taekwondo athletes. International SportMed Journal. 15(4).492-499.

23. SARSHIN, A.; FALLAHI, V.; FORBES, S.C.; RAHIMI, A.; KOOZEHCHIAN, M.S.; CANDOW, D.G.; KAVIANI, M.; KHALIFEH, S.N.; ABDOLLAHI, V.; NADERI, A. 2021. Short-term co-ingestion of creatine and sodium bicarbonate improves anaerobic performance in trained taekwondo athletes. Journal of the International Society of Sports Nutrition. 18(1):1-9.

https://doi.org/10.1186/s12970-021-00407-7
24. STAUFFER, K.; NAGLE, E.; GOSS, F.; ROBERTSON, R. 2015. Valoracion de la potencia anaerobica de jugadoras de basquetbol universitarias de primera division. Revista de Entrenamiento Deportivo. 29(4).

25. TASKIN, M.; AKKOYUNLU, Y. 2020. Effect of Anaerobic Power on Agility and Quickness in Male National Taekwondo Athletes. Kinesiologia Slovenica. 26(2):49-57.

26. TAYECH, A.; MEJRI, M.A.; CHAABENE, H.; CHAOUACHI, M.; BEHM, D.G.; CHAOUACHI, A. 2019. Test-retest reliability and criterion validity of a new taekwondo anaerobic intermittent kick test. Journal of Sports Medicine and Physical Fitness. 59(2):230237.

https://doi.org/10.23736/S0022-4707.18.08105-7

27. TAYECH, A.; MEJRI, M.A.; CHAOUACHI, M.; CHAABENE, H.; HAMBLI, M.; BRUGHELLI, M.; BEHM, D.G.; CHAOUACHI, A. 2020. Taekwondo Anaerobic Intermittent Kick Test: Discriminant Validity and an Update with the Gold-Standard Wingate Test. Journal of Human Kinetics. 71(1):229-242.

https://doi.org/10.2478/hukin-2019-0081

28. VASCONCELOS, B.B.; PROTZEN, G.V.; GALLIANO, L.M.; KIRK, C.; DEL VECCHIO, F.B. 2020. Effects of high-intensity interval training in combat sports: a systematic review with metaanalysis. Journal of Strength and Conditioning Research. 34(3):888-900.

https://doi.org/10.1519/JSC.0000000000003255 